\title{
Achieving Sharp Resonances in Metamaterials through Symmetry Breaking
}

\author{
V. A. Fedotov, N. Papasimakis and N. I. Zheludev \\ Optoelectronics Research Centre, University of Southampton, SO17 1BJ, UK \\ e-mail: vaf@phys.soton.ac.uk \\ M. Rose \\ School of Physics and Astronomy, University of Southampton, SO17 1BJ, UK \\ S. L. Prosvirnin \\ Institute of Radio Astronomy, National Academy of Science, Kharkov, 61002, Ukraine
}

\begin{abstract}
We report on the new way of achieving sharp transmission and reflection resonances in subwavelength structured artificial materials.
\end{abstract}

Here we report the first experiential results on a new type of planar metallic metamaterials, which shows exceptionally narrow transmission and reflection stop-bands. The development of structure with a large resonance quality factor and narrow resonant spectral band is crucially important for developing photonics metamaterials such as negative index media, polarization and frequency selective surfaces etc.

We achieve the high quality resonances by introducing a symmetry breaking in the shape of metal inclusions forming the metamaterial. The new type of metamaterial that we report here is formed by a periodic array of identical sub-wavelength planar metallic "particles", which are structured in the form of an asymmetrically split ring (ASR) and placed on a dielectric substrate (see Fig. 1a). The ASR-structure exhibits a very sharp resonant response with the quality factor at least one order of magnitude large than that typically encountered in the existing metamaterials and frequency selective surfaces. We show that the nature of the high-Q response can be associated with so-called closed or dark modes - anti-symmetric current modes excited in the ASR-particles, which are weakly coupled to free-space [1]. Coupling to such modes is usually forbidden unless the particles have a certain structural asymmetry. Manifestation of the resonant properties depends on the type of the symmetry breaking. For the case of asymmetrically split rings shown in Fig. 1a (a metallic ring split into 2 unequal parts) the metamaterial exhibits an ultra-narrow resonant reflection stopband near $5.5 \mathrm{GHz}$ (see Fig. 1b).

At this resonance, as shown in the inset to Fig. 1b, two parts of the particle are excited in anti-phase, which dramatically reduces coupling to free-space. "Dark mode" resonance is accompanied by two "conventional" resonances, where excitation of one part of the particle dominates the other (see Fig. 1b). We also found that if the symmetry breaking is achieved by dividing the ring along its diameter into two equal arcs with cuts of different size an ultra-narrow stop-band is seen in transmission near 5.5 GHz. Remarkably, in symmetrical case, i.e. when the arcs as well as cuts are equal, no high-Q resonances can be

(a)

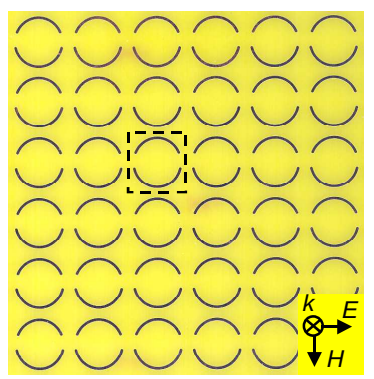

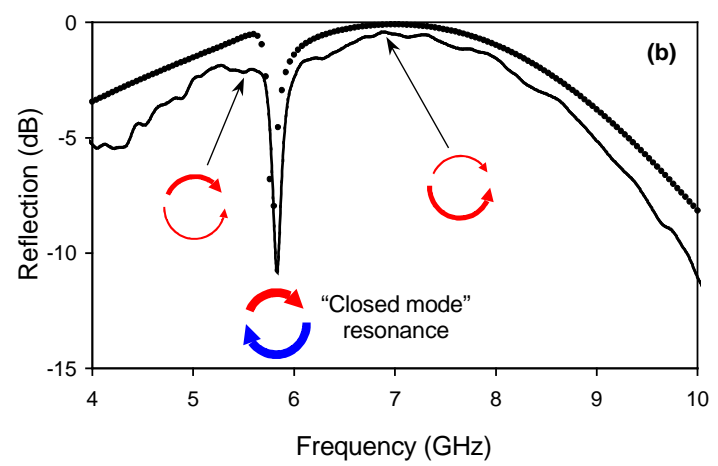

Frequency $(\mathrm{GHz})$

Fig. 1. (a) A fragment of metamaterial with asymmetrically structured particles (two unequal arcs), where dashed line box indicates a $15 \times 15 \mathrm{~mm}$ elementary translational cell of the structure. (b) Intensity of electromagnetic wave reflected from planar metamaterial: solid line - experiment, filled circles - theory. Insets present resonant current distributions in the asymmetric particles: arrows show instantaneous directions (phases) of the induced currents while thickness of the lines correspond to relative intensities of the currents. observed.

[1] S. Zouhdi, S. Prosvirnin, Advances in Electromagnetics of Complex Media and Metamaterials (Kluwer Academic Publishers, Netherlands, 2003), pp. 281-290 